\title{
O impacto das atividades remotas das ligas acadêmicas no ensino e na aprendizagem
}

\section{na graduação}

\author{
The impact of remote academic league activities on undergraduate teaching and learning \\ El impacto de las actividades de la liga académica remota en la enseñanza y el aprendizaje de
} pregrado

Fernanda Raquel Ribeiro Machado ORCID: https://orcid.org/0000-0002-6681-3413 Universidade de Itaúna, Brasil

E-mail: fernandarrm99@gmail.com

Regina Consolação dos Santos

ORCID: https://orcid.org/0000-0002-7393-3210 Universidade do Estado de Minas Gerais, Brasil Universidade Federal São João del-Rei, Brasil E-mail: regina.consolacao@uemg.br

Alysson Geraldo Mendonça ORCID: https://orcid.org/0000-0003-4090-1391 Universidade Federal dos Vales do Jequitinhonha e Mucuri, Brasil

E-mail: alysson_mendonca@hotmail.com

Fernanda Marcelino Rezende e Silva ORCID: https://orcid.org/0000-0003-2236-7009 Universidade do Estado de Minas Gerais, Brasil

E-mail: fernanda.silva@uemg.br

Thays Cristina Pereira Barbosa ORCID: https://orcid.org/0000-0003-1816-0662 Universidade do Estado de Minas Gerais, Brasil E-mail: thayscristina19@gmail.com

Caique Alves Rezende ORCID: https://orcid.org/0000-0003-0956-7321 Universidade do Estado de Minas Gerais, Brasil E-mail: caiquerezende25@gmail.com Matheus Fellipe Alves Justo ORCID: https://orcid.org/0000-0002-7924-351X Universidade do Estado de Minas Gerais, Brasil

E-mail: matheushtv@gmail.com

Cláudia Martins da Costa

ORCID: https://orcid.org/0000-0001-5904-6829 Universidade do Estado de Minas Gerais, Brasil

E-mail: Claudiacostamello.92@gmail.com

\begin{abstract}
Resumo
Introdução: A pandemia da COVID-19 levou o mundo a adotar medidas de contenção. O surgimento de novas práticas trouxe consigo também outras relações de convívio entre os universitários com a adoção de práticas emergentes e urgentes, o ensino EAD. Algumas ferramentas são importantes para o desenvolvimento da aprendizagem dos discentes, e interação entre docentes e discentes, são elas os centros acadêmicos e ligas acadêmicas. Objetivo: Avaliar o impacto das mudanças causadas pela pandemia nas atividades dos estudantes de graduação analisando a inserção da atividade remota na vida do estudante. Métodos: Uma revisão integrativa da literatura. O presente trabalho se norteou a partir da seguinte questão norteadora: "Qual é o impacto das atividades remotas das ligas acadêmicas no contexto do ensinoaprendizagem na graduação"? Resultados: Dos seis artigos selecionados, todos estão no idioma português e ambos apresentam uma abordagem do tipo relato de experiência, quatro deles foram publicados em 2020, enquanto os outros dois no ano de 2021. No Quadro 1, é apresentada a síntese dos estudos incluídos no trabalho para melhor visualização dos conteúdos e resultados. Conclusão: Grande relevância ressaltar que as ligas acadêmicas promovem uma elevação no potencial para a transformação do cenário dos centros universitários. Essa característica ficou mais evidente durante a pandemia pois a rotina de todos os estudantes e profissionais da área da saúde passou a se modificar.
\end{abstract}

Palavras-chave: Educação a distância; COVID-19; Formação universitária. 


\begin{abstract}
Introduction: A COVID-19 pandemic prompted the world to adopt containment measures. Or the emergence of new practices also brought with it other relationships of coexistence among university students with the adoption of emerging and urgent practices, or distance education. Some tools are important for the development of two-student learning and interaction between faculty and students, within academic centers and academic leagues. Objective: Evaluate the impact of the changes caused by the pandemic in the activities of undergraduate students, analyzing the insertion of remote activity in the student's life. Methods: An integrative literature review. The present work was guided by the following guiding question: "What is the impact of remote activities of academic leagues in the context of teaching-learning in undergraduate courses"? Results: The six selected articles are all in Portuguese and both present an experience reporttype approach, four of them were published in 2020, while the other two in 2021. Table 1 shows the harmony of the included studies at work for better visualization of content and results. Conclusion: It is very important to emphasize that academic leagues promote an increase in the potential for transforming the scenario of university centers. This characteristic was more evident during the pandemic, as the routine of all students and professionals in the health area began to change.
\end{abstract}

Keywords: Distance Education; COVID-19; University education.

\title{
Resumen
}

Introducción: Una pandemia de COVID-19 llevó al mundo a adoptar medidas de contención. O el surgimiento de nuevas prácticas trajo también consigo otras relaciones de convivencia entre estudiantes universitarios con la adopción de prácticas emergentes y urgentes, o educación a distancia. Algunas herramientas son importantes para el desarrollo del aprendizaje de dos estudiantes y la interacción entre profesores y estudiantes, dentro de los centros académicos y las ligas académicas. Objetivo: Evaluar el impacto de los cambios provocados por la pandemia en las actividades de los estudiantes de pregrado, analizando la inserción de la actividad remota en la vida del estudiante. Métodos: Una revisión integradora de la literatura. El presente trabajo estuvo guiado por la siguiente pregunta orientadora: "Cuál es el impacto de las actividades remotas de las ligas académicas en el contexto de la enseñanza-aprendizaje en los cursos de pregrado"? Resultados: Los seis artículos seleccionados están todos en portugués y ambos presentan un enfoque tipo informe de experiencia, cuatro de ellos fueron publicados en 2020, mientras que los otros dos en 2021. La Tabla 1 muestra la armonía de los estudios incluidos en el trabajo para una mejor visualización de contenido y resultados. Conclusión: Es muy importante destacar que las ligas académicas promueven un incremento en el potencial de transformación del escenario de los centros universitarios. Esta característica se hizo más evidente durante la pandemia, ya que la rutina de todos los estudiantes y profesionales del área de la salud comenzó a cambiar.

Palabras clave: Educación a distancia; COVID-19; Enseñanza superior.

\section{Introdução}

No ano de 2019, em Wuhan, China surgiu um novo vírus, denominado coronavírus ou SARS-COV-2, que causa uma síndrome respiratória aguda, grave, com alta capacidade de contaminação. Devido sua alta capacidade de contaminação, no ano de 2020 se tornou uma pandemia, com proporções catastróficas, no âmbito de saúde pública, ambiental e econômica. Até o mês de abril de 2021 a SARS-COV-2 causou 406.437 óbitos confirmados. E são atualmente 14.725.975 casos confirmados de contaminação (Brasil, 2021).

A pandemia causada pela referida doença altamente contagiosa, perdurou pelo final de 2019, pelo ano de 2020 e 2021. Fato este que levou o mundo a adotar medidas de contenção ao COVID-19, sendo elas o usam de máscara individual de proteção, uso de álcool 70\%, fechamento de comércio não essencial, e isolamento social. A partir da adoção destas medidas, novos hábitos como o home office e a suspensão das aulas nos espaços físicos se fez necessário, migrando as interações dos grupos universitários para os ambientes virtuais até então, muito utilizado pela EaD. O surgimento de novas práticas trouxe consigo também outras relações de convívio entre os universitários com a adoção de práticas emergentes e urgentes para lidar com a pandemia (Santana, 2020).

O ensino EAD, adotado então como nova modalidade de ensino no Brasil, tem sido desafiadora para docentes, discentes e instituições que até então operavam o processo de ensino aprendizagem em ensino presencial. Algumas ferramentas são importantes para o desenvolvimento da aprendizagem dos discentes, e interação entre docentes e discentes, são elas os centros acadêmicos e ligas acadêmicas. Dentro das universidades, os centros acadêmicos são formados por estudantes que, seguindo 
orientações de seus mestres professores, promovem atividades caracterizadas nos moldes do ensino, pesquisa e extensão, (Ferreira, et al., 2016).

Estes grupos estudantes em especifico as ligas acadêmicas desempenham papel importante, oferecem dentro do ensino, aulas online, discussões de casos, palestras, mini cursos, mesas redondas, aulas práticas para desenvolver o aprendizado e diminuir as dificuldades de conhecimento que possam ocorrer durante o período de estudo dos alunos (Cavalcante et al., 2018).

Diante disto no que se refere às pesquisas e desenvolvimento de conhecimento científico, as ligas organizam eventos científicos e viabilizam o desenvolvimento de estudos com publicação e apresentação de estudos, desenvolvidos por seus membros. Na extensão, transmitem ações na comunidade a fim de auxiliar a população em geral sobre diversos temas, trazendo um impacto social positivo (Rezende et al., 2021).

Muito se tem debatido sobre as experiências dos estudantes e as respostas ao ensino remoto, e, consequentemente, sobre os efetivos resultados (Almanar, 2020). Fatores como menor comprometimento de tempo, alta taxa de retenção, soluções de prática on-line de questões, sala de aula invertida e teleconferências são colocados em pauta dentro dos centros acadêmicos a fim de melhorar a qualidade do ensino e proporcionar maiores avanços no contexto atual. (Almanar, 2020; Chick et al., 2020).

Assim o presente estudo tem como objetivo realizar uma revisão bibliográfica para avaliar o impacto das mudanças causadas pela pandemia nas atividades dos estudantes de graduação analisando a inserção da atividade remota na vida do estudante.

\section{Metodologia}

O estudo foi realizado através de uma revisão integrativa da literatura. Ela permite a realização da síntese de pesquisas realizadas sobre um determinado tema ou questão de forma sistematizada, ordenada e abrangente. Além disso, ajuda na construção de um corpo de conhecimento para os pesquisadores (Ercole,2014).

A busca dos trabalhos foi realizada de forma online nas bases de dados: Biblioteca virtual em saúde (BDENF), Literatura Latino-Americana e do Caribe em Ciências da Saúde (LILACS), Scientific Electronic Library Online (SciELO) e Medical Literature Analysis and Retrieval System Online (MEDLINE) no período de março a abril de 2021.

Os descritores utilizados foram: "Educação à distância”, "COVID-19”, "Ensino Superior, conforme a classificação dos Descritores em Ciências da Saúde (DECS)". Já de acordo com o Medical Subject Headings (MESH), foram utilizados: Enseignement à distance, Education, Higher. Visto isso, foram usados os operadores booleanos "OR" e "AND" para dar sentido à busca. Além disso, utilizou-se o termo "liga acadêmica" (academic league) como palavra-chave para uma segunda busca independente, na biblioteca virtual "Google Scholar", devido a sua importância no contexto da pesquisa apesar de não ser contemplado no DeCS.

Os critérios de inclusão determinados foram: artigos completos, disponíveis na integra, publicados entre os anos de 2019 a 2021 e de idiomas: português, inglês e espanhol. Excluíram-se todos aqueles que não correspondiam à temática abordada. Foi realizada a leitura dos títulos e resumos como forma de identificação do tema e classificação dos estudos que se encaixavam no tema escolhido.

Após a busca e classificação, todos os artigos passaram pela leitura na integra de três pesquisadores para a seleção. Toda e qualquer discordância foi colocada em discussão para que todos chegassem a um consenso. Para a organização dos artigos um instrumento foi elaborado, contemplando os itens de identificação dos estudos: Autores, título, ano de publicação e abordagem metodológica.

O presente trabalho se norteou a partir da seguinte questão norteadora: "Qual é o impacto das atividades remotas das ligas acadêmicas no contexto do ensino-aprendizagem na graduação"? 
Figura 1. Fluxograma referente ao processo de seleção dos estudos.

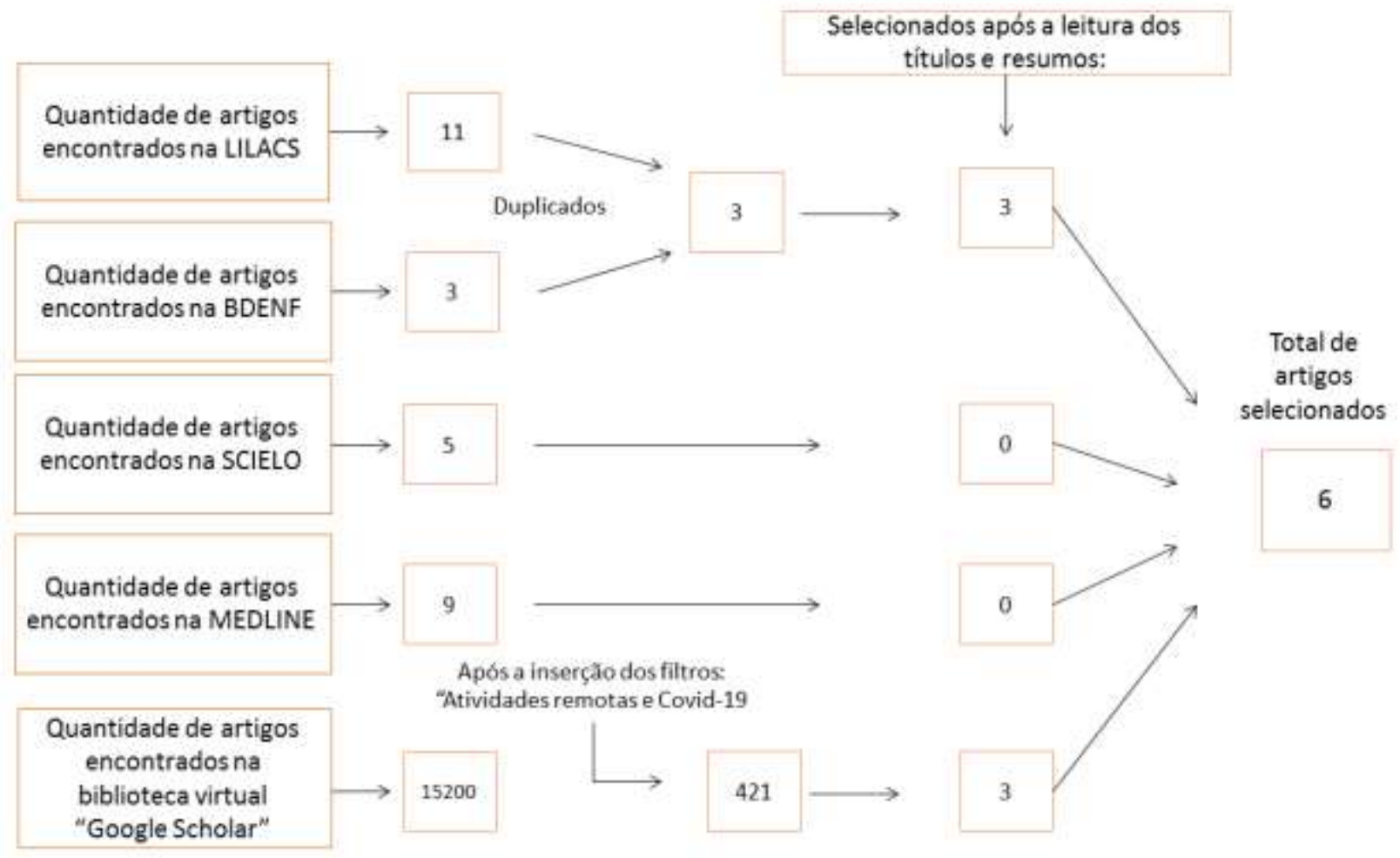

Fonte: Autores (2020).

\section{Resultados}

Dos seis artigos selecionados, todos estão no idioma português e ambos apresentam uma abordagem do tipo relato de experiência, quatro deles foram publicados em 2020, enquanto os outros dois no ano de 2021. No Quadro 1, é apresentada a síntese dos estudos incluídos no trabalho para melhor visualização dos conteúdos e resultados.

Quadro 1. Características dos estudos selecionados.

\begin{tabular}{|c|c|c|c|c|}
\hline Autores & Título & $\begin{array}{l}\text { Ano de } \\
\text { publicação }\end{array}$ & $\begin{array}{l}\text { Abordagem } \\
\text { metodológica }\end{array}$ & Revista \\
\hline $\begin{array}{l}\text { Bastos, Milena de Carvalho; } \\
\text { Canavarro, Danielle de } \\
\text { Andrade; Campos, Luana } \\
\text { Moura; Schulz, Renata da } \\
\text { Silva; Santos, Josely Bruce } \\
\text { dos; Santos, Claudenice } \\
\text { Ferreira dos. }\end{array}$ & $\begin{array}{l}\text { Ensino remoto emergencial na } \\
\text { graduação em enfermagem: relato } \\
\text { de experiência na covid-19 }\end{array}$ & 2020 & $\begin{array}{l}\text { Relato de } \\
\text { experiência }\end{array}$ & $\begin{array}{l}\text { Reme : Rev. Min. } \\
\text { Enferm }\end{array}$ \\
\hline 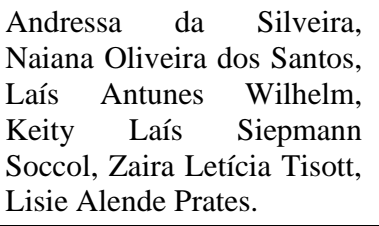 & $\begin{array}{l}\text { Estratégias e desafios do ensino } \\
\text { remoto na enfermagem }\end{array}$ & 2020 & $\begin{array}{l}\text { Relato de } \\
\text { Experiência }\end{array}$ & Enferm. Foco \\
\hline $\begin{array}{l}\text { Araújo, Agostinho Antônio } \\
\text { Cruz; Amaral, Jackeline } \\
\text { Vieira; Araujo Filho, } \\
\text { Augusto Cezar Antunes } \\
\text { de; Nunes, Benevina Maria } \\
\text { Vilar Teixeira }\end{array}$ & $\begin{array}{l}\text { O ensino de graduação em } \\
\text { enfermagem durante a pandemia } \\
\text { da COVID-19. }\end{array}$ & 2021 & $\begin{array}{l}\text { Relato de } \\
\text { Experiência }\end{array}$ & Revista Cuidarte \\
\hline
\end{tabular}




\begin{tabular}{|c|c|c|c|c|}
\hline $\begin{array}{lr}\text { Fábio Andrey da } & \text { Costa } \\
\text { Araújo1,André } & \text { Vajgel } \\
\text { Fernandes,Fernanda } & \text { Souto } \\
\text { Maior dos Santos,João Vitor } \\
\text { Gonçalves } & \text { do } \\
\text { Carmo,Matheus } & \text { Pereira } \\
\text { Frazão,Annanda } & \text { Hellen } \\
\text { Cadengue de Siqueira,José } \\
\text { Sandro Xavier } & \text { Duarte } \\
\text { Júnior,Hortência } & \text { Vitória } \\
\text { Silva Oliveira } & \\
\end{array}$ & $\begin{array}{l}\text { Liga Acadêmica de Cirurgia e } \\
\text { Traumatologia Bucomaxilofacial: } \\
\text { experiência duranteCOVID-19 }\end{array}$ & 2021 & $\begin{array}{ll}\text { Relato } & \mathrm{de} \\
\text { experiência } & \end{array}$ & $\begin{array}{l}\text { Revista de Extensão } \\
\text { da UPE }\end{array}$ \\
\hline $\begin{array}{l}\text { RaquelSantiago } \\
\text { Vitorino,Júlia Holandino da } \\
\text { Silva,Gabriela Ferreira dos } \\
\text { Anjos Araújo,Julie Ruiz } \\
\text { Lessa, Marcelle Braconnot } \\
\text { Fonseca Pires, Gabriela } \\
\text { Barbosa Pires dos Santos, } \\
\text { Vinícius Figueira Ramos, } \\
\text { Michelle Teixeira Teixeira, }\end{array}$ & $\begin{array}{l}\text { Experiências de uma Liga } \\
\text { Acadêmica de Nutrição e } \\
\text { Alimentação Materno Infantil: } \\
\text { Um Relato de Caso }\end{array}$ & 2020 & $\begin{array}{ll}\text { Relato } & \mathrm{de} \\
\text { experiência }\end{array}$ & $\begin{array}{l}\text { Semear- Revista de } \\
\text { Alimentação, } \\
\text { Nutrição e Saúde }\end{array}$ \\
\hline $\begin{array}{l}\text { Crissia Pitanga Malta1, } \\
\text { Willian Alves dos Santos, } \\
\text { Paulo Gustavo Aguiar de } \\
\text { Oliveira, Larissa Parada } \\
\text { Leite, Andréa de Andrade } \\
\text { Dias Soares, Barbara } \\
\text { Andrade de Lima, Karen } \\
\text { Sanae Takehara Vieira, } \\
\text { Kathleen Serrão Pena, Maria } \\
\text { Eduarda Morgado da Silva, } \\
\text { Marcelo Imbroinise } \\
\text { Bittencourt }\end{array}$ & $\begin{array}{l}\text { Atuação da liga acadêmica de } \\
\text { cardiologia durante a pandemia da } \\
\text { Covid-19 e as implicações na } \\
\text { educação médica: relato de } \\
\text { experiência }\end{array}$ & 2020 & $\begin{array}{l}\text { Relato de } \\
\text { experiência }\end{array}$ & $\begin{array}{l}\text { Revista Eletrônica } \\
\text { Acervo Saúde } \\
/ \\
\text { Elect } \\
\text { ronic Journal } \\
\text { Collection Health }\end{array}$ \\
\hline
\end{tabular}

Fonte: Autores (2020).

\section{Discussão}

Com a pandemia da COVID-19 e o consequente isolamento social, diversos impactos foram impostos à sociedade, determinando mudanças de hábitos e rotinas (Soares, 2020). Os indivíduos acometidos pela doença produzem uma grande quantidade partículas virais após poucos dias de infecção, o que contribui para a disseminação, que ocorre por meio do contato, bem como pelo compartilhamento de objetos contaminados. Como um fator agravante, a transmissão pode ocorrer poucos dias após a infecção, mesmo antes do aparecimento dos sintomas da doença, entre indivíduos, o que aumenta a taxa de transmissão da doença por conta da ausência de isolamento e da manutenção das atividades cotidianas (Heyman, 2020; Wu et al., 2020).

Nesse contexto, métodos baseados no ensino remoto, ferramenta importante na difusão de conhecimento, foram propostos para o ensino remoto nas atividades acadêmicas remotas emergenciais. Em curto prazo, fez-se necessária a regulamentação de atividades extracurriculares, pesquisas, extensão e trabalhos de conclusão de curso (TCC). Em médio prazo, as universidades foram obrigadas a preparar a estrutura necessária para as atividades acadêmicas, como aulas on-line, promover a inclusão digital de professores e implementar políticas de assistência estudantil que fornecessem as ferramentas necessárias para a adesão dos discentes.

As Ligas Acadêmicas são caracterizadas como organizações formadas por núcleos estudantis sem fins lucrativos, que desenvolvem com seus membros atividades didáticas, científicas, culturais e sociais, que acercam uma determinada área. Essas atividades visam o crescimento e progresso dos estudantes. As Ligas são administradas pelos próprios alunos o que exige uma articulação interpessoal entre seu grupo formador e criando-se habilidades gerenciais, com a orientação de um ou mais docentes (Pontes, 2017). 
Essas Ligas são formadas respeitando o princípio constitucional que rege o Ensino Superior que é a indissociabilidade entre ensino, pesquisa e extensão, conhecido como tripé universitário. Essas organizações podem ser compostas de alunos de apenas um curso ou de cursos diversos, sempre respeitando o estatuto que rege a sua formação (Silva et al.,2018).

No que diz respeito à relevância no âmbito social, as ligas acadêmicas promovem benefícios diretos e indiretos à comunidade. Quanto aos benefícios diretos, convém ressaltar o desenvolvimento de diversas ações educativas visando a conscientização da população acerca de problemas referentes à saúde, além da realização de campanhas de doação de sangue e de arrecadação de alimentos, materiais de limpeza e de higiene. Já o benefício indireto à sociedade corresponde ao melhor preparo dos profissionais para que, no futuro, estes possam entender os determinantes do processo saúde-doença, responder às necessidades sociais, além de saber como atuar e promover a educação em saúde, refletindo positivamente na comunidade.

Nesse contexto, observa-se que a atuação das ligas acadêmicas está em consonância à Política Nacional de Promoção da Saúde. Essa política coloca a promoção de saúde como uma estratégia indispensável ao Sistema Único de Saúde (SUS), uma vez que contribui para ações que possibilitem responder às necessidades sociais, promovendo a qualidade de vida e reduzindo riscos e vulnerabilidades (Silva et al.,2018).

Esses grupos desempenharam um importante papel durante a pandemia causada pela COVID-19, tanto no momento de distanciamento social quanto nos momentos de crise econômica que estamos enfrentando. As Ligas desenvolvem diversas atividades durante esses períodos laboriosos que a sociedade enfrenta, reforçando essas organizações num contexto social (Yang et al.,2019).

As ligas foram orientadas a realizar tanto ensino como ações na comunidade remotamente, por meio de transmissões ao vivo e/ou vídeos educativos on-line em plataformas como o YouTube, Google Meet, Zoom, WhatsApp, Instagram, dentre outras. As atividades on-line das ligas iniciaram em junho de 2020. Assim existiu a possibilidade de realização de ações voltadas para membros da liga, ou para grupos específicos, como estudantes do ensino fundamental e médio, mulheres, gestantes, crianças de determinadas instituições e seguidores das redes sociais das ligas, por exemplo (Oliveira et al.,2020).

Umas das principais dificuldades em promover aulas remotas online é a inacessibilidade, em especial por famílias de baixa renda. Essa condição sugere um escasso investimento em políticas públicas de inclusão social, sendo um momento oportuno para se investir em políticas que garantam acesso à internet e dispositivos tecnológicos a todos (Joyce et al., 2020)

Independente das dificuldades e obstáculos que o ensino remoto traz, como as dificuldades de internet boa para todos e a supressão do contato social, é notório como podemos levar vários ensinamentos desse período para o futuro. Com certeza, mesmo com o retorno as atividades presenciais, o ensino remoto continuará sendo utilizado pelas ligas. Esse novo modelo pode levar as ligas a disporem de novas possibilidades de sustentar o tripé, maior alcance na sociedade e de um maior intercâmbio de informações em todo país (Santana, 2020).

Várias instituições de ensino superior optaram pela continuidade de seu ano letivo sob a perspectiva do ensino remoto, porém, em muitos casos essa mudança abrupta teve sérios problemas de adaptação. As ligas acadêmicas possuem um papel fundamental no atual momento pois, a estas, foi dada a oportunidade de continuidade de execução de planejamento, caso se adaptassem a realidade de ensino remoto (Fernandes et al., 2020). Com a dificuldade causada pelo espalhamento do coronavírus e as restrições governamentais quanto as aglomerações, fez-se necessário que as pessoas elaborassem formas de se manterem unidas e conectadas e formassem novas maneiras de manterem os laços. Popularizou-se assim as Web conferências, Vídeos Chamadas e Lives.

Com a criação de grupos com ligas de diferentes instituições e de diferentes estados pode-se ter uma troca de experiências de qual estratégia estava funcionando num estado, como estavam fazendo para dar continuidade as suas atividades, quais canais estavam dando resultado e pode-se fechar parcerias com outros seguimentos do ensino ampliando dessa forma, as abordagens a população em geral. Segundo estudo3, esse trabalho pode induzir o crescimento pessoal tanto nos quesitos da sua 
formação profissional como na sua capacidade geral de gestão. Os alunos podem aplicar na prática seus conhecimentos adquiridos desde o início da graduação em suas atividades desenvolvidas para a comunidade acadêmica e social.

\section{Conclusão}

É de grande relevância ressaltar que as ligas acadêmicas promovem uma elevação no potencial para a transformação do cenário dos centros universitários, muito atrelado as suas ações de ensino, pesquisa e extensão dando assistência e realizando transformação social tanto dentro das universidades como também na sociedade em que está inserida. As LAs contribuem para a formação acadêmica não somente da Enfermagem, porém suas ações unem todas as outras áreas do conhecimento. Dessa forma a liga contribui para formar profissionais que valorizem seus aspectos éticos e morais sempre colaborando para o trabalho Inter e multidisciplinar através de espaços democráticos e humanitários.

Essa característica ficou mais evidente durante a pandemia pois a rotina de todos os estudantes e profissionais da área da saúde passou a se modificar. A busca por novos moldes para a distribuição da informação e da pratica, foram os divisores de águas para as ligas. Talvez a dificuldade de se ter contato físico aflorou outras perspectivas dentro do curso, trazendo mais conteúdo e diversidade de metodologias para dentro das universidades.

Com este estudo esperamos que instituições de ensino privado e particular incentivem a oficina de ligas acadêmicas como meio de ensinamento e diversidade no meio estudantil independente da classe social, religião, gênero e valores culturais.

\section{Referências}

Almanar, M. A. The shifting of face to face learning to distance learning during the pandemic Covid-19. Globish: An English-Indonesian Journal for English, Education, and Culture. 9(2)1-8, 2020.

Brasil, Ministério da Saúde., Painel Coronavírus https://covid.saude.gov.br/.

Caique, A. R. “A importância da liga acadêmica de saúde materno-infantil em tempos de covid-19: relato de experiência”, International Journal of Development Research, 11, (02), 44721-44723

Cavalcante, A. S. P. et al. As ligas acadêmicas na área da saúde: lacunas doconhecimento na produção científica brasileira. Revista Brasileira de Educação Médica, 42(1), 199-206.

Chick, R., et al. Using technology to maintain the education of residents during the COVID-19 pandemic. J Surg Educ. 77(4):729-32, 2020.

Estadão De São Paulo. Avanços tecnológicos impulsionados pela pandemia. https://summitsaude.estadao.com.br/tecnologia/avancos-tecnologicos impulsionados-pela-pandemia.

Ercole, F. F.; Melo, L. S.; \& Alcoforado, C. L. G. C. Revisão integrativa versus revisão sistemática. REME, 18. http://www.dx.doi.org/10.5935/14152762.20140001

Fernandes, S. M., et al. O ensino a distância no Brasil: alguns apontamentos. Research, Society and Development, 9(2), 19.

Ferreira, I. G. et al. Atividades extracurriculares e formação médica: diversidade e flexibilidade curricular. IJHE-Interdisciplinary Journal of Health Education, 1(2).

Heymann, D. L.; \& Shindo, N. COVID-19: what is next for public health? The Lancet, 395(10224), 542-545.

Joye, C. R et al. Educação a Distância ou Atividade Educacional Remota Emergencial: em busca do elo perdido da educação escolar em tempos de COVID-19. Research, Society and Development, 9(7), e521974299-e521974299.

Machado J. A., \& Pauna, H. Distance learning and telemedicine in the area of Otorhinolaryngology: lessons in times of pandemic. Braz J Otorhinolaryngol. $86(3): 271-2,2020$.

Oliveira, C. P. et al. Liga Acadêmica de Psicologia da Saúde: compromisso social e formativo. ANALECTA -Centro de Ensino Superior de Juiz de Fora, Juiz de Fora, 5(5)

Pêgo-Fernandes, P. M.; \& Mariani, A. W. O ensino médico além da graduação: ligas acadêmicas. Diagn Tratamento, 16(2), 50-1.

Pontes, S. M.; \& Torreao, L. A. Influência da participação dos alunos em ligas acadêmicas na escolha da especialidade para o Programa de Residência Médica Bahia 2017. Revista de Medicina. 98(3):16. 
Research, Society and Development, v. 10, n. 12, e550101220695, 2021

(CC BY 4.0) | ISSN 2525-3409 | DOI: http://dx.doi.org/10.33448/rsd-v10i12.20695

Santana, C. Pedagogias das conexões: ensinar e aprender na sociedade digital blended. In: Educação em rede: construindo uma ecologia para a cultura digital, $6(1)$.

Silva, D. P. et al. Proposição, fundação, implantação e consolidação de uma liga acadêmica. Revista de Enfermagem UFPE on line. 12(5):1486, 2018.

Soares, C. S. A.; \& Fonseca, C. L. R. Atenção primáriaà saúde em tempos de pandemia.Journal of Management \& Primary Health Care, 12, 1-11.

Wong, H. Y. F. et al. Frequency and Distribution of Chest Radiographic Findings in COVID-19 Positive Patients. Radiology, 201160.

Wu, F. et al. A new coronavirus associated with human respiratory disease in China. Nature, 579(7798), 265-269.

Yang, G. Y. et al. Liga de Anatomia Aplicada (LAA): as Múltiplas Perspectivas sobre Participar de uma Liga Acadêmica. Rev. bras. educ. med. $43(1): 80$ 86.

Zhu, N. et al. A novel coronavirus from patients with pneumonia in China, 2019. New England Journal of Medicine. 